\title{
THE METAPHYSICAL FOUNDATIONS OF PHYSICS
}

Carlos ROMERo

carlos.a.romero.c@icloud.com

Recent naturalistic approaches to metaphysics emphasize the need for the discipline to establish a direct connection with contemporary science - including, but not limited to, physics. In his contribution to this issue, Gustavo E. Romero characterizes this stance as follows:

Metaphysics cannot, and certainly should not, try to offer a final, ultimate worldview. It should evolve with science and in accordance with what the special sciences have to tell us about the world. And from there it should move on to elaborate always provisional general truths about whatever is thought to exist. (p. 74)

But can philosophical reflection and critique and systematization of the most fundamental concepts and theories also contribute to the foundations of physics? This special issue of Crítica is devoted to this question.

In what follows, I will provide a summary of each of the papers in this special issue.

\section{Primitive Beables Are Not Local Ontology: On the Relation between Primitive Ontology and Local Beables}

Valia Allori's paper is an excellent introduction to the current debate on the ontology of quantum mechanics. Its main aim is to clarify the difference between two approaches in the field: the approach in terms of beables, championed by Bell, and the approach in terms of primitive ontology, championed by Allori, among others. Let us first clarify the context of these proposals, guided by Allori's exposition of the matter.

In response to the measurement problem of quantum mechanics, empirical theories were designed to replace the standard theory with one that does not require the unclear notion of measurement. The standard theory requires that notion in order to formulate the 
measurement postulate, which claims, roughly, that upon measurement a quantum system will reduce its state - which, typically, is one of superposition - to an eigenstate of the measured observable. Measurement, then, is what the standard theory uses to eliminate macroscopic superpositions, which are never observed.

Three alternatives stand out: the pilot wave theory, the spontaneous localization family of theories, and the many-worlds theory. All these explain away observable superpositions without mentioning measurement in their fundamental postulates, albeit they do so in radically different ways. As Allori notes, the metaphysics of quantum mechanics today is mainly concerned with understanding the ontology of these theories.

Some philosophies of contemporary quantum theories simply refuse to inquire more about ontology, or even to think that the formalism represents something ontological: instrumentalist and quantum Bayesianism, for example. On the other side we find realist approaches, those that take quantum theories to represent something physically real, not mere instruments for predicting experiences.

Perhaps the first rigorous realist approach to non-standard quantum theories is Bell's, with his notion of local beables. A beable contrasts with an observable in that it does not need to be observed in order to be, and is to be radically distinguished from purely formal additions to the theory: a beable of a theory is a part of its mathematics that describes the ontology, what there is in the world. Bell urged physicists to understand the local beables of their theories, these being "the mathematical counterparts in the theory to real events at definite places and times in the real world" (p. 17). Then, the quantum wave function may be a beable (depending on the theory and its interpretation), but it is not a local beable.

Among exactly formulated quantum theories, we find different approaches, derived from the answers to these three questions:

1. What does the wave function represent?

2. Does the wave function collapse?

3. Is the basic ontology local in three-dimensional, ordinary space?

The three questions are obviously highly interrelated, so we should not think of them as independent dimensions of the theory space.

A positive answer to the second question is given by the spontaneous collapse family of theories, like the GRW and CSL theories; 
the answer to the first and third question depends on the exact version and interpretation. The many-worlds theory, in contrast, rejects collapse: the terms in a superposition represent the many 'branches' of the emergent multiverse, and then, the basic ontology is not in ordinary 3D space, but in configuration space; it remains unclear what exactly does the wave function represent. Bohmian mechanics does not need collapse, either, as it postulates both a wave of probability (represented by the wave function), which can be superposed, and particles — which always have a definite location. The particles are 'guided by' the wave in a sense to be further interpreted and, at least on the classical interpretation, do live in 3D space. Depending on how these theories are interpreted, we can specify their beables. And, again depending on interpretation, those beables are going to be local or not.

One way to interpret those theories is to claim that their fundamental equations specify their beables; in particular, the fundamental ontological postulate is that of the wave function. The wave function realists think that the wave function represents, at least in Bohmian and spontaneous collapse approaches, something real and fundamental in the world. But, as the wave function is defined on the highdimensional configuration space and not on our ordinary 3D space, and as it can enter into superposed states, the fundamental ontology is not local in $3 D$ space, according to the wave function realists. It is, rather, local in configuration space: wave function realists take the wave function as the beable of quantum theories that is local in the fundamental space, the space of configurations.

A debate on the proper sense of locality for quantum theory ensues. One may insist that the relevant sense of locality is locality in $3 \mathrm{D}$ space. We then have a schism in the realist camp, with wave function realists on the one side, and localists, as Allori calls them, on the other.

In the localist camp we find the primitivists about quantum-states, the multi-field theorists, the spacetime-state realists, and the primitive ontologists. Again, these views are realist approaches; they also think that the fundamental ontology is local in 3D space. Allori explains some reasons why a quantum realist should be a localist.

As said above, Allori's aim in the paper is to distinguish her primitive ontology approach from the approach in terms of local beables. The claim is that "a primitive ontology is a special type of local beable" (p. 22), and the point is to specify the exact differences.

According to Allori (p. 27), the primitive ontology has three roles: 
1) A metaphysical role: it defines what materially exists at the microscopic level in three-dimensional space;

2) An explanatory role: it explains macroscopic phenomena dynamically and constructively;

3) A physical role: it defines symmetries as invariances of its law of evolution.

The first aspect is that, according to the primitive ontologists, we have an a priori metaphysical hypothesis as to the nature of matter, that results from a simplicity constraint. The hypothesis is that matter is three-dimensional, since that is what we observe. The simplicity constraint restricts the theories, but is also "fundamentally tied to simplicity of explanation" (p. 23), says Allori, in that it urges to "retain the compositional and dynamical explanation of the phenomena typical of the classical theories" (p. 23). That is, macroscopic matter is explained by its microscopic components (the compositional aspect) and their motions (the dynamic aspect). Those microscopic material components are the primitive ontology of the theory, which, then, satisfy the metaphysical and explanatory roles.

If the observed phenomena is explained in terms of the dynamics of its material micro-constituents, what role does the wave function play in the explanation? Primitive ontologists think of it as having a nomological role: the wave function is part of the law of evolution of the primitive ontology. So, it represents something objective: it is a beable; but not something material. According to Allori, it is local in the sense of existing in spacetime. Then, primitive ontologists subdivide local beables: some are primitive, representing matter; some are nomological, representing part of the laws of evolution of matter.

The last role of the primitive ontology is related to symmetries. Given the primitive ontology as the result of the constraint of simplicity on ontology, the laws that explain the dynamics of the phenomena are a result of another constraint of simplicity, this time mediated by the symmetries, which are thought of as the invariances for the law for the primitive ontology. According to Allori, "Making the symmetries a business of the primitive ontology allows us to explain why the wave function transforms the way it does under symmetry transformations in order to make the theory invariant" (p. 27).

Against those who think of the wave function as a non-local beable, like quantum state primitivists, Allori notes that the dynamical 
equations for the local beables can be written in many forms, each appealing to very different mathematical objects: density matrices, for example, instead of wave functions. Those formulations are physically equivalent, as they reproduce the very same histories of the local beables. Allori thinks that this, and the absence of any criteria to take any particular formulation as the metaphysically fundamental one, should prevent us to reify the wave function and similar objects. She concludes that "the most straightforward attitude towards these entities [...] is to think of them not as representing some unfamiliar, mysterious nonlocal beable, but as part of the laws" (p. 30). She also provides another argument against taking the wave function as a beable, based on symmetry considerations.

Allori's paper provides a resourceful overview of the current debate, and helps clarify the region of theory space neighboring her own primitive ontology approach.

\section{Calculation, Bookkeeping, Representation, and Explanation: A Parable}

Tim Maudlin's paper presents simple-looking yet profound morals in his characteristic proficiency of prose.

We begin with the well-known problem of the ontology of the quantum wave function. Maudlin notes that some popular ways of phrasing the fundamental question betray philosophical confusions. For example, the question: 'Is the wave function real?' fails to separate questions pertaining to the ontology of mathematics and others pertaining to the ontology of physics. This is a reminder of the importance of distinguishing the mathematical apparatus from what it is supposed to represent in the physical world. So, Maudlin opts for this phrasing of the ontological question: "What, if anything, does the wave function represent about the physical system to which it is ascribed?" (p. 46).

He also briefly considers the question, that has gained wide attention recently, whether the wave function is ontic or epistemic. To be 'ontic' means that the wave function represents objective probabilities of the system; it is 'epistemic' if it represents the credal state of a certain agent. Quantum Bayesianism is a recent incarnation of the latter thought, where the credences that quantum theory is supposed to recommend are about what expected experiences an agent will have. According to Maudlin, this kind of instrumentalism leads to the puzzling conception that physics is about predicting experiences, 
rather than providing explanations of why the natural world works the way it does. In contrast,

The physicist — or should we say natural philosopher?wants $[\ldots]$ an accurate account of the nature of the physical world and would not be terribly surprised if that account also allows one to make accurate and reliable predictions, although that is just a side benefit. (p. 49)

This leads us to the question whether the standard quantummechanical description of physical reality is 'complete', or whether it needs some additional apparatus. While Everettians and objective collapse theorists answer that it is, Bohmians answer that it is not, and posit local beables — usually, but not necessarily, particle configurations - to complete the picture.

In contrast, the quantum Bayesians believe that the quantum description is not only incomplete, but that it is "part of a prescription", says Maudlin, "about the degrees of subjective credence an agent has or should have" (p. 50). And this leads us to the question of how could the wave function "be so effective as a part of a predictive mechanism" (p. 50), given that it does not represent anything objective. This question, in turn, leads Maudlin to Putnam's famed no miracles argument for realism, which we can compress into Putnam's sentence that "The positive argument for realism is that it is the only philosophy that doesn't make the success of science a miracle" (p. 50).

Maudlin is unconvinced that the no miracles argument represents the situation correctly. He notes that Ptolemaic astronomy, Newtonian gravitation, and Maxwellian electrodynamics were empirically successful and well-developed theories, even though their ontological claims were fundamentally misguided. But Maudlin refuses to believe that their empirical success was miraculous in any reasonable sense. He's not interested to develop, in this paper, a detailed diagnosis of what has gone wrong with Putnam's argument; rather, his paper is focused on two narrower but still clearly related questions: What is it to regard a mathematical apparatus as referring to something physical? How can we explain the empirical success of a mathematical formalism?

Maudlin's presentation of the issues is cleverly made through a fable, which I recommend for the enjoyment of the reader. In it, Maudlin uses the case of negative and positive numbers to impart lessons on how mathematical representations of physical reality can 
have both representational and non-representational parts in the same theory, while both parts are governed by purely mathematical facts. These mathematical facts can be used to simplify the calculation with the use of mathematical fictions - in Maudlin's example, negative numbers in the royal treasury. With this example, Maudlin suggests a two-step explanation of empirical success: first, a "physical argument', deploying mathematical representations that are taken as representations of something physical, and second, a "purely mathematical argument' that certain calculations are guaranteed to give identical (or approximately identical) results, even if they use different intermediate steps and then different mathematical objects. He uses this two-step explanation to provide an explanation of how Newtonian physics can be scientifically explainable, even though its central terms do not refer to anything physically real, and points out that the same could be argued for Feynman's proposal of negative probabilities and the gauge freedom in Maxwell's electrodynamics.

At this point, Maudlin advocates for his previously defended view that a physical theory needs not only the mathematical formalism, but what he calls a commentary: a specification of both the fundamental and derivative ontology of the theory, as well as a specification of how the mathematics represents each element of the ontology. $\mathrm{He}$ notes that the same mathematical apparatus may be connected to different ontologies through different commentaries.

Maudlin ends his paper further illustrating his view with the case of virtual particles, and noting how his framework can be applied to the ontology of the wave function. Again, he recommends ontological clarity, and notes several arguments in favor of the wave function being a representation of something physical, though noting that the question remains of what exactly.

The consequences of Maudlin's approach are wide-reaching. It shows how to be a scientific realist without relying on Putnam's argument. It also promotes, in the spirit of clarity, the thinking of physical theories as essentially including an ontological interpretation, which requires a clean separation of the mathematics that play a representational role from the mathematics that are only 'gauge' degrees of freedom.

\section{Black Hole Philosophy}

Gustavo E. Romero's paper is a delightful tour through some of the physical and philosophical conundrums that black holes raise. These 
puzzling entities were used to be thought as highly theoretical, but as Romero notes, the direct observation of gravitational waves by the LIGO experiment in 2016 and other merger events, and the direct imaging of the shadow of a supermassive black hole, have dispelled almost every doubt about their existence. The questions now are about the implications of their existence.

Romero begins the paper providing the basic definitions in the field. For the mathematical representation of the spacetime, we have a pseudo-Riemannian manifold that is four-dimensional, infinitely differentiable, and real. On top of this, we have a metric field, that basically specifies spacetime intervals in infinitesimal regions of the manifold. From the metric we have the Ricci curvature tensor and the scalar curvature, which specify the local curvature of spacetime. Apart from these, to formulate Einstein's equations we need the cosmological and the Einstein gravitational constants, and a tensor representing the energy-momentum of all physical fields at a region. Basically, Einstein's equations tell us that the curvature at each point of spacetime is determined by the energy-momentum at that point. Furthermore, we need the definition of the causal structure of spacetime: basically, a definition of the arrow of time in terms of the local light cone structure (specified continuously throughout the manifold), defining which events can be causally affected by, and can causally affect, which others.

Black holes are regions of spacetime, so they are characterized by the curvature. Notably, though the events inside a black hole are all determined by past events, none of them is causally connected to events in the global external future of spacetime: because the curvature is such that, once inside their boundary - the event horizon-, it would take superluminal speed to get out, nothing can get out of a black hole region. So, a spacetime contains a black hole if at least one of its regions is causally disconnected from the global future.

Romero's paper is so wide-ranging that I could not dare to summarize all of its content. In the hope of providing an invitation to its study, however, I will showcase some of the topics that it presents.

One of the first philosophical issues that Romero deals with is determinism. If we conceive of the doctrine as the thesis that all events are given, GR is a deterministic theory. Note the absence of any epistemic or causal concepts in the formulation. In particular, note how it differs from the traditional, Laplacian formulation, according to which the world is deterministic if a specification of a state, together with the laws that govern the universe, determines 
the complete history of the universe. To this formulation, Romero objects that it conflates uniqueness-of-solution theorems with reality, because the world is not mathematical. Further, he notes that black holes provide another reason to break the determinism-predictability link, because they contain Cauchy horizons - basically, hypersurfaces where the initial value problems for the equations of the matter fields fail to be well-defined even in the presence of a complete specification of initial conditions.

Romero also provides arguments against presentism based on the existence of black holes and of gravitational waves. Roughly, presentism is the thesis that only the present is real; it is opposed by both eternalism, the thesis that the past, present and future are equally real, and by the growing block theory, that only the past and present, but not the future, are real. (The other logically possible theory opposed to eternalism would be that only the future is real, but no one has dared to propose such a view!)

Romero claims that General Relativity is incompatible with presentism, which is all the more evident in the presence of black holes. One argument is a variant of the classical argument from the frame-relativity of simultaneity - basically, because simultaneity is frame-dependent and because the ontology cannot be framedependent, presentism is falsified. Possible escapes from the argument are examined and rejected.

A novel argument is also offered (p. 102):

P1. There are gravitational waves.

P2. Gravitational waves have non-zero Weyl curvature.

P3. Non-zero Weyl curvature is only possible in 4 or more dimensions.

$\mathrm{P} 4$. Presentism is incompatible with a 4-dimensional world.

Then, presentism is false.

Premise 1 is defended on empirical grounds, while premises 2 and 3 follow from the mathematics of general relativity. The only remaining premise is the fourth. However, as Romero notes, presentism is incompatible with there being objects extended in time, having temporal parts, because presentism simply is the thesis that there are no future or past events.

Novel and very interesting arguments in favor of spacetime substantivalism also make an appearance. They are all based on currently 
confirmed features of spacetime and the fact that those features can only be had by material entities. Here's the first argument (p. 106):

P1. Only material entities can be heated.

P2. Spacetime can be heated.

Therefore, spacetime is a material entity.

The first premise follows from general ontological principles about materiality. The second premise follows from the thermodynamics of black holes, which Romero also overviews in a previous section. The thought is that the event horizon of a black hole has a temperature, and because a black hole is nothing but a region of spacetime, spacetime does have a temperature: it can be heated when something falls through the horizon of the black hole.

A similar argument is given (p. 106):

P1. Spacetime has entropy.

P2. Only things with a microstructure can have entropy.

Therefore, spacetime has a microstructure.

P3. If something has a microstructure, it must exist.

Thus, spacetime exists.

And again, we know the first premise to be true because the event horizon does have entropy. The second premise is true by the definition of entropy, and the third relates again to general ontological principles about materiality. A third argument for substantivalism, offered by the late Mario Bunge, is similar but based on the materiality and reality of gravitational waves, which are ripples of spacetime. Romero also notes that Einstein himself realized the materiality of spacetime, and the fact that it can exist without any other material entity, after his debate with de Sitter.

The final issue that I will mention is the ontological status of black holes. Romero notes that, according to the best developed physics of today, the world is made up of quantum fields on spacetime. These fields seem to be mereologically simple and not ontologically dependent on anything else. Particles, as excitations of the fields, are properties of the fields (conceived of as in Heil's ontology of modes of substances). The question is how do black holes fit in this scheme. 
According to Romero, black holes can interact with fields, but they do not seem to be formed by them. They are material, for sure - at least on Romero's Bungean criteria for materiality: having energy. Black holes "are fully material because they can do work on other material systems" (p. 122). They are, as we saw at the beginning, regions of spacetime with particular curvatures. This curvature is also what explains their capacity to affect other physical systems; so, black holes exert works through the metric of spacetime, and spacetime also exerts work from other derivations of its metric (like gravitational waves). Then, Romero proposes to take black holes not as substances, but as modes of substances, again in the sense of properties: "Spacetime can be in different ways. One way is what we call a black hole" (p. 122).

As announced, I did not talk about many of the topics that Romero surveys - from black hole thermodynamics, to the arrow of time or supertasks, to the problem usually termed 'the black hole information paradox', to the nature of spacetime singularities (Romero is an anti-realist about them), to the topic of black holes in the FLRW model of cosmology. Undoubtedly, Romero's paper repays closer study, and will become the standard reference of the metaphysics of black holes for years to come.

I hope that this special issue will not only contribute to the specialized debates in the philosophy of physics, but also attract interest from the broader audience of Crítica.

Finally, I want to present my gratitude to each of the authors, reviewers, and administrative personnel who made this special issue possible. Their contributions and cooperation represent a shining of light amidst the darkness of the toughest health issue that humanity has faced in the recent years. 